Grunberg A, Juschenko A, Santopietro R, Cintorino M, Tosi P, Syrjanen S. Oral contraceptives are not an independent risk factor for cervical intraepithelial neoplasia or high-risk human papillomavirus infections. Anticancer Res. 2006; 26: 4729-40.

\section{Anatomical assessment of ventricular septum in relation to the aortic valve of bovine heart}

In recent decades, thromboembolic complications, ruptures, stenoses, and calcifications of the aortic heart valves cause morbidity and mortality of human patient. These complications need valvular replacement or correction with the natural valve, either mechanical or biological ${ }^{1}$. Implantation of the valve is necessary for the correction of the diseased human valve. These implanted valves may be mechanical, may be of porcine origin or bovine pericardial origin. Now-a-days, in Bangladesh porcine aortic heart valves and mechanical valves have been using randomly in the cardiac patients, although these are expensive for the poor patients. Thinking with this view an attempt had been made for implanting the bovine aortic heart valve to the poor patients. In an initial attempt, a study had been carried out with Bangladesh Heart Research Association, and it was found that the orifice diameter of indigenous bovine aortic heart valve ranged from 21 to $35 \mathrm{~mm}^{2}$, which was similar to that of human being. Because of that, conservative techniques have been progressively used in patients with valvopathies in a number of centers. Thus, the interest in the morphology of cardiac valves, which during the 60's and 70's was limited to the pathological features due to the large number of valve replacements, was extended. The more accurate knowledge of normal anatomy began to play an important role in the success of valvoplasties, as many decisions during surgery are based on the visual assessment of the changes. Detailed knowledge of the anatomical characteristics of the aortic valve should improve the understanding of its anatomy and help much to obtain the better results in conservative procedures and in this way promoting return to anatomical and functional normality. This precise knowledge also defines some details of the architecture of the aortic valve that are necessary for the development and manufacture of the prostheses. In addition to performing an anatomical assessment, they analyzed some linear variables in each cusp and documented the presence of some fenestrations close to the commissural attachments. Jatene et al. ${ }^{4}$ observed that the aortic valve annulus did not show a perfect circumference, with some variations in the measurements of the annulus, in the cusps and in the relation with the ventricular septum.

A total of 32 bovine heart specimens were collected from the slaughter-house of Mymensingh District by adopting an aseptic measure. After rinsing away the blood, the hearts were kept in the balanced isotonic saline solution until dissection in the laboratory. The aortic heart valves of the collected bovine heart were harvested by dissection. This study included the position of the ventricular septum in relation to the aortic heart valve. Using a standard mathematical measuring scale and a cotton thread, the left coronary cusp (LC), right coronary cusp (RC), and noncoronary cusp (NC) were measured (Jatne et al., 1999) ${ }^{7}$ using following parameters: the distance between the two septal extremities, one in between RC-LC commissure (A), another in between RC-NC commissure (B), distance between the septum and the NC-LC commissure (C) and aortic diameter (D) were measured as showing in Figure 1.

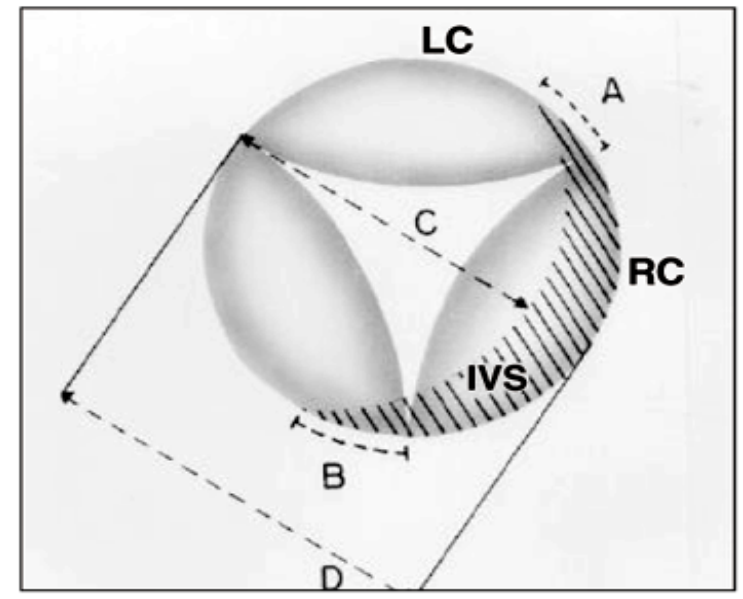

Figure 1: A Sketch showing the position of the interventricular septum (IVS) in relation to the aortic valve. LC-left coronary; RCright coronary; NC-noncoronary; A- distance between the septal extremity and the RC-LC commissure; B-distance between the septal extremity and the RC-NC commissure; C-distance between septum and the NC-LC commissure; D-aortic diameter

The position of the interventricular septum (IVS) in regard to the aortic valve annulus and its structures, the mean values of the $\mathrm{A}, \mathrm{B}$ and $\mathrm{C}$ measurements were $13.81 \pm 1.60 \mathrm{~mm} ; 14.72 \pm 2.14 \mathrm{~mm}$; and $17.66 \pm 2.86 \mathrm{~mm}$ respectively. There were no statistically significant differences in regard to sex and age. The mean aortic diameter (measure D) was $23.28 \pm 2.85 \mathrm{~mm}$, and there were no significant sexual and age differences. The measure $\mathrm{D}$, however, was smaller in hearts of animals under the age of 3 years, and it showed a progressive increase with age but statistically that was insignificant $(\mathrm{p}>0.05)$. Therefore, the ratio $\mathrm{C} / \mathrm{D} \mathrm{x}$ 
32 showed mean values of $24.53 \pm 1.84 \mathrm{~mm}$, and it was larger in the hearts of animals under the age of 3 years (Table 1 ).

Table 1: Mean values of the position of the ventricular septum and its relation with the aortic valve (in $\mathrm{mm})(\mathrm{n}=32)$

\begin{tabular}{cccc}
\hline \multirow{2}{*}{ Measures } & Mean value & \multicolumn{2}{c}{ Sex } \\
\cline { 3 - 4 } & & Male & Female \\
\hline A & 13.81 & 13.56 & 14.06 \\
B & 14.72 & 14.13 & 15.31 \\
C & 17.66 & 17.50 & 17.81 \\
D & 23.28 & 22.69 & 23.88 \\
Ratio C/D & 24.53 & 25.30 & 23.75 \\
\hline
\end{tabular}

The mean aortic diameter (D) of the bovine heart was $23.28 \pm 2.85 \mathrm{~mm}$ and there were no significant sexual and age differences in the present study. The aortic diameter was smaller in hearts of animals under the age of 3 years, and it had showed a progressive increment with age but statistically it was insignificant $(p>0.05)$. Jatene et al. ${ }^{4}$ also reported the similar observations in human heart and found that the mean diameter of the aorta was $21.8 \pm 3.6 \mathrm{~mm}$, and there were no significant sexual or racial differences, but the diameter increased progressively with the increase in age. They also added that the aortic heart valve annulus did not show a perfect circumference with some variations in the measurements of the annulus, in the cusps and in the relation with the ventricular septum. In the present study, it was also revealed that the ratio $\mathrm{C} / \mathrm{D}$ was higher in hearts of animals $<3$ years of age and progressively decreased with the increase in age. This may suggest that perhaps left ventricular outflow tract (LVOT) shows a trend to proportionally decrease its area in relation to the aortic diameter with increasing age.

The author acknowledges the financial supports provided by FnF Pharmaceutical Ltd. Company, Bangladesh. The author also grateful to Bangladesh Heart Research Association and Bangladesh Agricultural University for collaborating research and logistic assistance.

\section{Md. Nazrul Islam}

Department of Anatomy and Histology Faculty of Veterinary and Animal Science Sylhet Agricultural University, Sylhet, Bangladesh. e-mail:mnislam58@yahoo.com

\section{References}

1. Braunwald E. A textbook of cardiovascular medicine. $4^{\text {th }}$ edn. Philadelphia, WB Saunders Co, 1992, pp 104353.
2. Khan SR, Islam MN. Studies on the prospect of bioprostheses by bovine aortic valve for human use. Bangladesh Med Res Counc Bull. 1991; 17: 75-80.

3. Fontes RD, Bonassa J, Jatene AD (1991). Configuração esférica do aparelho valvar aórtico. Arq Bras Cardiol. 1991; 57: 385-88.

4. Jatene MB, Monteiro R, Guimarães MH, Veronezi S C, Koike MK, Jatene FB, Jatene AD. (1999). Anatomical study of 100 healthy human hearts. Arq Bras Cardiol. 1991; 73: 1 .

\section{Regulation of serum total protein and choles- terol level in cold-exposed rat}

Several factors involved in the regulation of serum protein and cholesterol levels of humans as well as rodents. Cold exposure is the major sympathetic and environmental stimuli regulating metabolic functions ${ }^{1}$. After prolonged exposure of rats to cold, a sustained increase in systolic blood pressure occurs, giving rise to a cold-induced model of hypertension $^{2}$. Activation of the sympathetic nervous system is thought to contribute to the development of hypertension in cold-exposed rats ${ }^{3}$. Sympathetic input to the kidney increases the release of renin, which in turn contributes to the production of Angiotensin II. The sympathetic output from the hypothalamus in response to cold exposure has been shown to regulate glycogen metabolism, degradation of glycogen to glucose and appeared in the higher glucose concentration in the serum. Moreover, cold-induced thermoregulation is associated with an increase in lipid metabolism ${ }^{4,5}$. Triglyceride degradation after sympathetic stimulation is predominantly observed. The resulting higher free fatty acids in the serum interact with serum binding protein like bovine serum albumin. It is speculated that cold exposure stimulates the accumulation of serum protein as well as cholesterol because of the constriction of blood vessels by higher blood pressure. The prolonged exposure may result vasodilatation regulating homeostasis of these constituents. Therefore, the present study has been undertaken whether cold exposure is involved in the regulation of serum constituents like protein and cholesterol.

Male rats weighing 200 to $260 \mathrm{~g}$ were used. They were housed in the cages at ambient temperature and given free access to laboratory foods and water. In the day of experiment, cold exposure (4$8^{\circ} \mathrm{C}$ ) were given to the different groups of rats in the cold chamber for $30 \mathrm{~min}, 1$ hour, 2 hour and 4 hour with full aeration and with free access of water. After cold exposure treatment, the rats were immediately anesthetized with diethyl ether and 